PROCEEDINGS OF THE

AMERICAN MATHEMATICAL SOCIETY

Volume 133, Number 11, Pages 3421-3427

S 0002-9939(05)08264-X

Article electronically published on June 20, 2005

\title{
CONTINUOUS SELECTIONS AND FIXED POINTS OF MULTI-VALUED MAPPINGS ON NONCOMPACT OR NONMETRIZABLE SPACES
}

\author{
LAI-JIU LIN, NGAI-CHING WONG, AND ZENN-TSUEN YU \\ (Communicated by Jonathan M. Borwein)
}

\begin{abstract}
In this paper, we obtain several new continuous selection theorems for multi-valued mappings on completely regular spaces and fixed point theorems for multi-valued maps on nonmetrizable spaces. They, in particular, provide a partial solution of a conjecture of X. Wu.
\end{abstract}

\section{INTRODUCTION}

In [4 5], Browder first used a continuous selection theorem to prove the FanBrowder fixed point theorem. Later, Yannelis and N. D. Prabhakar [17, Ben-ElMechaiekh [2, 3], Ding, Kim and Tan [8], Horvath [11, Wu [16, 15, Park [12, 13, and many others, established several continuous selection theorems with applications. We note that in all the continuous selection theorems studied by the above authors, the multi-valued maps are defined on a compact or paracompact space. In [18, $\mathrm{Yu}$ and Lin studied continuous selections of multi-valued mappings defined on noncompact spaces, but they assume some kind of coercivity conditions instead.

In this paper, we establish a continuous selection theorem for a multi-valued map defined on a completely regular topological space. We do not assume the compactness of its domain.

In the second part of this paper, we discuss collectively fixed points of lower semicontinuous multi-valued maps. Recently, many authors studied fixed point theorems of lower semicontinuous multi-valued maps; see for example [14, 6, 15, 1]. In particular, Wu established the following one.

Theorem 1.1 ([15]). Let $X$ be a nonempty subset of a Hausdorff locally convex topological vector space, let $D$ be a nonempty compact metrizable subset of $X$, and let $T: X \rightarrow 2^{D}$ be a multi-valued mapping with the following properties:

(a) $T(x)$ is a nonempty closed convex set for each $x$ in $X$;

(b) $T$ is lower semicontinuous.

Then there exists a point $\bar{x}$ in $D$ such that $\bar{x} \in T(\bar{x})$.

Wu conjectured in [15] that the conclusion of Theorem 1.1 remains true even if the metrizability condition of $D$ is dropped. In this paper, we shall use the

Received by the editors July 17, 2003.

2000 Mathematics Subject Classification. Primary 54C65, 46H10, 54H25.

Key words and phrases. Multi-valued mappings, continuous selections, fixed points.

(C)2005 American Mathematical Society 
approximate continuous selection theorem of Deutsch and Kenderov [7] (see also [20]) to establish an approximate fixed point theorem for a sub-lower semicontinuous multi-valued map. This gives rise to a partial solution of the conjecture of $\mathrm{Wu}$ [15]. We shall also provide a simple proof of a Himmelberg type collectively fixed point theorem. We remark that our results differ from the approximate fixed point theorem recently established by Park [13.

We would like to thank the referee for many helpful suggestions on improving the presentation and the bibliography in this paper.

\section{Preliminaries}

Let $X$ and $Y$ be topological spaces. A multi-valued map $T: X \rightarrow 2^{Y}$ is a map from $X$ into the power set $2^{Y}$ of $Y$. Let $T^{-1}: Y \rightarrow 2^{X}$ be defined by the condition that $x \in T^{-1} y$ if and only if $y \in T(x)$. Recall that

(a) $T$ is said to be closed if its graph $G_{r}(T)=\{(x, y): x \in X, y \in T(x)\}$ is closed in the product space $X \times Y$;

(b) $T$ is said to be upper semicontinuous (in short, u.s.c.) at $x$ if for every open set $V$ in $Y$ with $T(x) \subset V$, there exists a neighborhood $W(x)$ of $x$ such that $T(W(x)) \subset V ; T$ is said to be u.s.c. on $X$ if $T$ is u.s.c. at every point of $X$;

(c) $T$ is said to be lower semicontinuous (in short, l.s.c.) at $x$ if for every open neighborhood $V(y)$ of every $y$ in $T(x)$, there exists a neighborhood $W(x)$ of $x$ such that $T(u) \cap V(y) \neq \emptyset$ for all $u$ in $W(x)$; $T$ is said to be l.s.c. on $X$ if $T$ is l.s.c. at every point of $X$;

(d) in case $Y$ is a topological linear space, $T$ is said to be sub-lower semicontinuous (see, e.g., 20]) at an $x$ in $X$ if for each neighborhood $V$ of 0 in $Y$, there is a $z$ in $T(x)$ and a neighborhood $U(x)$ of $x$ in $X$ such that $z \in T(y)+V$ for each $y$ in $U(x)$; $T$ is said to be sub-lower semicontinuous on $X$ if $T$ is sub-lower semicontinuous at every point of $X$. It is plain that if $T$ is lower semicontinuous at $x$, then $T$ is sub-lower semicontinuous at $x$.

The following lemmas are needed in this paper.

Lemma 2.1 (Deutsch and Kenderov 7]). Let $X$ be a paracompact topological space, let $Y$ be a locally convex topological linear space, and let $F: X \rightarrow 2^{Y}$. Then $F$ is sub-lower semicontinuous if and only if for each neighborhood $V$ of 0 in $Y$, there is a continuous function $f: X \rightarrow Y$ such that $f(x) \in F(x)+V$ for each $x$ in $X$.

Lemma 2.2 (Yuan [19]). Let $X$ be a topological space, let $Y$ be a nonempty subset of a topological vector space with a base $\mathcal{B}$ for the zero neighborhoods, and let $F$ : $X \rightarrow 2^{Y}$. For each $V$ in $\mathcal{B}$, define $F_{V}: X \rightarrow 2^{Y}$ by

$$
F_{V}(x)=(F(x)+V) \cap Y, \quad \forall x \in X .
$$

Write $\bar{y} \in \bar{F}(\bar{x})$ if $(\bar{x}, \bar{y}) \in \overline{G_{r} F}$. Then for any $\bar{x}$ in $X$ and $\bar{y}$ in $Y$, we have

$$
\bar{y} \in \bar{F}(\bar{x}) \quad \text { whenever } \quad \bar{y} \in \bigcap_{V \in \mathcal{B}} \overline{F_{V}}(\bar{x}) .
$$

Lemma 2.3 (Himmelberg [10]). Let $X$ be a nonempty convex subset of a locally convex topological vector space. Let $T: X \rightarrow 2^{X}$ be a u.s.c. multi-valued map 
with nonempty closed convex values such that $T(X)=\bigcup_{x \in X} T(x)$ is contained in a compact subset of $X$. Then there exists an $\bar{x}$ in $X$ such that $\bar{x} \in T(\bar{x})$.

Lemma 2.4 (Granas [9]; see also Ding, Kim and Tan [8]). Let D be a nonempty compact subset of a topological vector space. Then the convex hull co $D$ of $D$ is $\sigma$-compact and hence is paracompact.

\section{Continuous selection theorems}

Note that the set $S^{-1}(y)=\{x \in X: y \in S(x)\}$ below can have empty interior for some $y$ in $K$.

Theorem 3.1. Let $X$ be a completely regular space and let $K$ be a nonempty subset of a Hausdorff topological vector space E. Assume a multi-valued function $S: X \longrightarrow 2^{K}$ satisfies the following conditions:

(a) For each $x$ in $X$, the set $S(x)$ is convex.

(b) $X=\bigcup\left\{\operatorname{int} S^{-1}(y): y \in K\right\}$.

Then for any compact subset $F$ of $X$ there is an open dense subset $U$ of $X$ containing $F$ such that $S$ has a continuous selection $f: U \rightarrow K$, that is, $f(x) \in S(x)$ for all $x$ in $U$.

Proof. By assumption (b), there are finitely many points $y_{1}, \ldots, y_{n}$ in $K$ such that

$$
F \subseteq \operatorname{int} S^{-1}\left(y_{1}\right) \cup \cdots \cup \operatorname{int} S^{-1}\left(y_{n}\right) .
$$

For each $k=1, \ldots, n$ and $x$ in $F \cap \operatorname{int} S^{-1}\left(y_{k}\right)$, there is a continuous function $g_{x}$ on $X$ such that $0 \leq g_{x} \leq 1, g_{x}(x)=1$ and $g_{x}$ vanishes outside int $S^{-1}\left(y_{k}\right)$. By the compactness of $F$, there are finitely many $g_{x}$ such that for every point in $F$ at least one of them assumes a value not less than $1 / 2$. Summing them in an appropriate way, we will have nonnegative continuous functions $g_{1}, \ldots, g_{n}$ on $X$ such that $g_{k}$ vanishes outside int $S^{-1}\left(y_{k}\right)$, and $\sum_{k=1}^{n} g_{k}(x) \geq 1 / 2$ for all $x$ in $F$. Let $V=\left\{x \in X: \sum_{k=1}^{n} g_{k}(x)>1 / 3\right\}$. Set $f_{j}(x)=g_{j}(x) / \sum_{k=1}^{n} g_{k}(x)$ on $V$, and $f_{j}(x)=3 g_{j}(x)$ on $X \backslash V$. Define a continuous function $f_{V}: X \longrightarrow E$ by

$$
f_{V}(x)=\sum_{k=1}^{n} f_{k}(x) y_{k}, \quad \forall x \in X .
$$

For each $x$ in $V$ and for each $k$ with $f_{k}(x) \neq 0$, we have $x \in \operatorname{int} S^{-1}\left(y_{k}\right)$. Hence, $y_{k} \in S(x)$. Consequently, $f_{V}(x) \in \operatorname{co}(S(x))=S(x) \subseteq K$ for all $x$ in $V$. In other words, the restriction of $f_{V}$ to $V$ gives rise to a continuous selection of $S$ on the open set $V$ which contains $F$.

Denote by

$$
\begin{aligned}
& \mathcal{W}=\left\{\left(f_{W}, W\right): \text { where } W \text { is an open subset of } X \text { containing } F\right. \text { and } \\
& \left.\qquad f_{W}: W \rightarrow K \text { gives rise to a continuous selection of } S \text { on } W\right\} .
\end{aligned}
$$

Then $\mathcal{W}$ is not empty as $\left(f_{V}, V\right) \in \mathcal{W}$. Order $\mathcal{W}$ by extension and we get a nonempty partially ordered set. In other words, $\left(f_{W}, W\right) \leq\left(f_{V}, V\right)$ if $W \subseteq V$ and $f_{\left.V\right|_{W}}=f_{W}$. Applying Zorn's Lemma, we get a maximal element $\left(f_{U}, U\right)$ of $\mathcal{W}$.

The last step is to verify that $U$ is dense in $X$. Suppose not and there were an $x$ in $X$ outside the closure of $U$. Let $x \in \operatorname{int} S^{-1}(y)$ for some $y$ in $K$. By setting $f_{\left.\right|_{W}} \equiv y$, we get a continuous selection of $S$ on an open neighborhood $W$ of $x$ 
disjoint from $U$ by restriction. Then the union $f_{U \cup W}: U \cup W \longrightarrow K$ defined in a natural way provides a contradiction to the maximality of $\left(f_{U}, U\right)$.

We call a topological space $X$ residually paracompact if for every open dense subset $U$ of $X$ the complement $X \backslash U$ is paracompact.

Theorem 3.2. In addition to conditions (a) and (b) in Theorem 3.1, if we assume further that

(c) $X$ is residually paracompact,

then there is a continuous function $f: X \rightarrow K$ such that $f(x) \in S(x)$ for all $x$ in $X$.

Proof. It follows from Theorem 3.1 that there is a continuous function $f_{U}: U \longrightarrow K$ defined on an open dense subset $U$ of $X$ with $f_{U}(x) \in S(x)$ for all $x$ in $U$. For each $z$ in $X \backslash U$, there is a $y$ in $K$ such that $z \in$ int $S^{-1}(y)$ by condition (b). By setting $f_{\mid W_{z}} \equiv y$ we get a continuous selection of $S$ on an open neighborhood $W_{z}$ of $z$. The paracompactness of $X \backslash U$ ensures it has a locally finite covering by open sets in $X$, each of which is contained in some $W_{z}$. Adding one more open set $U$, we have a locally finite open covering of $X$. This provides us with a family $\left\{g_{\lambda}\right\}_{\lambda}$ of nonzero continuous functions from $X$ into $[0,1]$ dominated by the open sets $W_{z}$ and $U$ such that $g_{\lambda}(x)=0$ for all but finitely many $\lambda$ 's and $\sum_{\lambda} g_{\lambda}(x)=1$ for all $x$ in $X$. If $g_{\lambda}$ vanishes outside $U$, we set $f_{\lambda}=f_{U}$. Otherwise, we fix a choice of $z$ such that $g_{\lambda}$ vanishes outside $W_{z}$, and set $f_{\lambda}=f_{W_{z}}$. Define $f: X \longrightarrow K$ by

$$
f(x)=\sum_{\lambda} g_{\lambda}(x) f_{\lambda}(x), \quad \forall x \in X .
$$

For each $x$ in $X$, only finitely many $g_{\lambda}(x)$ 's are nonzero in the sum, and the nonzero terms give rise to a convex combination of points in the convex set $S(x)$. Thus $f(x) \in S(x)$ for all $x$ in $X$.

It is easy to see that the following corollary follows from Theorem 3.1.

Corollary 3.3. The conclusion of Theorem 3.1 remains true if conditions (a) and (b) are replaced by

(a)' for each $x$ in $X$, the set $S(x)$ is a nonempty convex set;

(b)' for each $y$ in $K$, the set $S^{-1}(y)$ is open.

Remark 3.4. Corollary 3.3 implies Theorem 3.1 .

Proof. Let $T: X \rightarrow 2^{K}$ be defined by

$$
T(x)=\left\{y \in K: x \in \operatorname{int} S^{-1}(y)\right\} .
$$

Then $T^{-1}(y)=\operatorname{int} S^{-1}(y)$ is open for each $y$ in $K$. By (b), for each $x$ in $X$, there exists $y$ in $K$ such that $x \in \operatorname{int} S^{-1}(y)$. Therefore $y \in T(x) \neq \emptyset$ for each $x$ in $X$. Let $H: X \rightarrow 2^{K}$ be defined by $H(x)=\operatorname{co} T(x)$. Then $H(x)$ is nonempty for each $x$ in $X$, and $H^{-1}(y)$ is open for each $y$ in $K$. By Corollary 3.3, there is an open dense subset $U$ of $X$, containing any but a fixed compact set $D$, and there is a continuous function $f: U \rightarrow K$ such that $f(x) \in H(x)=\operatorname{co} T(x) \subset S(x)$ for all $x$ in $U$. 


\section{FiXed POINT ThEOREMS}

Theorem 4.1. For each $i$ in a nonempty index set $I$, let $X_{i}$ be a nonempty convex subset of a Hausdorff locally convex topological vector space $E_{i}$, and let $D_{i}$ be a compact subset of $X_{i}$. Let $X=\prod_{i \in I} X_{i}$ be the product space. Let $F_{i}: X \rightarrow$ $2^{D_{i}}$ be sub-lower semicontinuous with nonempty convex values. Then for every neighborhood $V_{i}$ of 0 in $E_{i}$, there exists a point $\bar{x}_{V}=\left(x_{V_{i}}\right)$ in $D=\prod_{i \in I} D_{i}$ such that $\left(\bar{x}_{V_{i}}+V_{i}\right) \cap F_{i}\left(\bar{x}_{V}\right) \neq \emptyset$ for all $i$ in $I$.

Proof. Let $V_{i}$ be a neighborhood of zero in $E_{i}$ for each $i$ in $I$. Fix any $i$ in $I$. There exists an absolutely convex neighborhood $W_{i}$ of 0 such that $W_{i} \subset V_{i}$. Note that $D$ is a compact subset of $X$. By Lemma 2.4 co $D$ is a paracompact subset of $X$. Since $F_{i}: X \rightarrow 2^{D_{i}}$ is a sub-lower semicontinuous multi-valued map with nonempty convex values, by Lemma2.1 there exists a continuous function $f_{i}$ : co $D \rightarrow D_{i}$ such that

$$
f_{i}(x) \in\left(F_{i}(x)+W_{i}\right) \cap D_{i} \text { for each } x \in \operatorname{co} D .
$$

Define $f: \operatorname{co} D \rightarrow D$ by $f(x)=\prod_{i \in I} f_{i}(x)$ for $x$ in co $D$. By the Himmelberg fixed point theorem (Lemma 2.3), there exists an $\bar{x}_{V}=\left(\bar{x}_{V_{i}}\right)_{i \in I}$ in co $D$ such that $\bar{x}_{V}=f\left(\bar{x}_{V}\right)=\prod_{i \in I} f_{i}\left(\bar{x}_{V}\right)$. That is, $\bar{x}_{V_{i}}=f_{i}\left(\bar{x}_{V}\right) \in\left(F_{i}\left(\bar{x}_{V}\right)+W_{i}\right) \cap D_{i}$. Thus, $\bar{x}_{V_{i}} \in D_{i}$ and $\left(\bar{x}_{V_{i}}+W_{i}\right) \cap F_{i}\left(\bar{x}_{V}\right) \neq \emptyset$ for all $i$ in $I$. Since $W_{i} \subset V_{i}$, we have $\left(\bar{x}_{i}+V_{i}\right) \cap F_{i}\left(\bar{x}_{V}\right) \neq \emptyset$ for all $i$ in $I$.

Theorem 4.2. Suppose in Theorem 4.1 we assume further that for each $x=$ $\left(\bar{x}_{i}\right)_{i \in I} \in X$, its coordinates $x_{i} \notin \overline{F_{i}}(x) \backslash F_{i}(x)$ for all $i$ in $I$. Then there exists a point $\bar{x}=\left(\bar{x}_{i}\right)_{i \in I} \in D=\prod_{i \in I} D_{i}$ such that $\bar{x}_{i} \in F_{i}(\bar{x})$ for each $i$ in $I$.

Proof. For each $i$ in $I$, let $\mathcal{B}_{i}$ be the collection of all absolutely convex open neighborhoods of zero in $E_{i}$ and $\mathcal{B}=\prod_{i \in I} \mathcal{B}_{i}$. Given any $V=\prod_{i \in I} V_{i}$ in $\mathcal{B}$, let $Q_{V}=\left\{x \in D: x_{i} \in \overline{F_{V_{i}}}(x)\right.$ for all $i$ in $\left.I\right\}$. Then $Q_{V}$ is a nonempty closed subset of $D$ for each $V$ in $\mathcal{B}$ by Theorem 4.1 Let $\left\{V^{(1)}, \cdots, V^{(n)}\right\}$ be any finite subset of $\mathcal{B}$. Write $V^{(i)}=\prod_{j \in I} V_{j}^{(i)}$, where $V_{j}^{(i)} \in \mathcal{B}_{j}$ for each $i=1, \cdots, n$. Let $V^{\prime}=\prod_{j \in I}\left(\bigcap_{i=1}^{n} V_{j}^{(i)}\right) \in \mathcal{B}$. Clearly, $\emptyset \neq Q_{V^{\prime}} \subseteq \bigcap_{i=1}^{n} Q_{V^{(i)}}$. Therefore, the family $\left\{Q_{V}: V \in \mathcal{B}\right\}$ has the finite intersection property. Since $Q_{V} \subset D$ for all $V$ in $\mathcal{B}$ and $D$ is compact, $\bigcap_{V \in \mathcal{B}} Q_{V} \neq \emptyset$. Let $\bar{x}=\left(\bar{x}_{i}\right)_{i \in I} \in \bigcap_{V \in \mathcal{B}} Q_{V}$. Then $\bar{x}_{i} \in \overline{F_{V_{i}}}(\bar{x})$ for all $i$ in $I$ and all $V_{i}$ in $\mathcal{B}_{i}$, i.e., $\bar{x}_{i} \in \bigcap_{V_{i} \in \mathcal{B}_{i}} \overline{F_{V_{i}}}(\bar{x})$ for all $i$ in $I$. It follows from Lemma 2.2 that $\bar{x}_{i} \in \bar{F}_{i}(\bar{x})$ for all $i$ in $I$. By assumption, $\bar{x}_{i} \in F_{i}(\bar{x})$ for all $i$ in $I$.

We remark that if $F_{i}$ is closed, then $x_{i} \notin \overline{F_{i}}(x) \backslash F_{i}(x)$ for each $x=\left(x_{i}\right)_{i \in I}$ in $X$. As a special case of Theorem 4.2 we have the following collectively Himmelberg type fixed point theorem.

Corollary 4.3. For each $i$ in a nonempty index set $I$, let $X_{i}$ be a nonempty convex subset of a locally convex topological vector space $E_{i}$, let $D_{i}$ be a nonempty compact subset of $X_{i}$, and let $f_{i}: X=\prod_{i \in I} X_{i} \rightarrow D_{i}$ be a continuous function. Then there exists $\bar{x}=\left(\bar{x}_{i}\right)_{i \in I} \in D=\prod_{i \in I} D_{i}$ such that $\bar{x}_{i}=f_{i}(\bar{x})$ for each $i$ in $I$.

If the index set $I$ is a singleton, then Theorem 4.2 reduces to the following corollary, which provides a partial solution to a conjecture of $\mathrm{Wu}$ [15. 
Corollary 4.4. Let $X$ be a nonempty convex subset of a locally convex topological vector space $E$, let $D$ be a nonempty compact subset of $X$, and let $F: X \rightarrow 2^{D}$ be sub-lower semicontinuous with nonempty convex values. Suppose $x \notin \bar{F}(x) \backslash F(x)$ for each $x$ in $X$. Then there exists a point $\bar{x}$ in $D$ such that $\bar{x} \in F(\bar{x})$.

By Theorem 4.1, we have the following almost fixed point theorem.

Corollary 4.5. The conclusions of Theorems 4.1 and 4.2 remain valid if the condition " $F_{i}: X \rightarrow 2^{D_{i}}$ is sub-lower semicontinuous for each $i$ in $I$ " is replaced by " $F_{i}^{-1}\left(y_{i}\right)$ is open for each $y_{i}$ in $D_{i}$ and each $i$ in $I$."

Finally we remark that in case $I$ is a singleton, Theorem 4.1 provides a different result from [12, Theorem 3].

\section{REFERENCES}

[1] R. Agarwal and D. O'Regan, Fixed point theory for maps with lower semicontinuous selections and equilibrium theory for abstract economies, J. Nonlinear and Convex Analysis, 2 (2001), 31-46. MR1828157 (2002m:49025)

[2] H. Ben-El-Mechaiekh, The coincidence problem for compositions of set-valued maps, Bull. Austral Math. Soc., 41 (1990), 421-434. MR1071044(91h:47060)

[3] H. Ben-El-Mechaiekh, Fixed points for compact set-valued maps, Questions Answers Gen. Topology, 10(1992), 153-156. MF,1180474

[4] F. E. Browder, A new generation of the Schauder fixed point theorem, Math. Ann., 174 (1967), 285-290. MR0223944 (36:6991)

[5] F. E. Browder, The fixed point theory of multi-valued mappings in topological vector spaces, Math. Ann., 177 (1968), 283-301. MR0229101 (37:4679)

[6] P. Cubiotti, Some remarks on fixed points of lower semicontinuous multifunctions, J. Math. Anal. Appl., 174 (1993), 407-412. MR1215621 (94c:47081)

[7] F. Deutsch and P. Kenderov, Continuous selections and approximate selection for set-valued mappings and applications to metric projections, SIAM J. Math. Anal., 14 (1983), 185-194. MR.0686245 (84c:54026)

[8] X. P. Ding, W. K. Kim and K. K. Tan, A selection theorem and its applications, Bull. Austral. Math. Soc., 46 (1992), 205-212. MR1183778 (93g:47072)

[9] A. Granas, Points fixes pour les applications compactes: espaces de Lefschetz et la théorie de l'indice, Séminaire de Mathématiques Supérieures 68, Presses de l'Université de Montréal, Montreal, Que., 1980. MR0569745 (81i:55002)

[10] C. J. Himmelberg, Fixed points of compact multifunctions, J. Math. Anal. Appl., 38 (1972), 205-207. MF0303368 (46:2505)

[11] C. D. Horvath, Extension and selection theorems in topological vector spaces with a generalized convexity structure, Ann. Fac. Sci., Toulouse 2, (1993), 253-269. MR1253391 (94i:54043)

[12] S. Park, Continuous selection theorems in generalized convex spaces, Numer. Funct. Anal. Optim., 25 (1999), 567-583. MR.1704961 (2000e:54014)

[13] S. Park, The Knaster-Kuratowski-Mazurkiewicz Theorem and almost fixed points, Top. Methods in Nonlinear Anal., 16 (2000), 195-200. MR1805047 (2001m:47118)

[14] G. Tian, Fixed point theorems for mappings with noncompact and nonconvex domains, $J$. Math. Anal. Appl., 158 (1991), 161-167. MR1113407 (92e:47110)

[15] X. Wu, A new fixed point theorem and its applications, Proc. Amer. Math. Soc., 125 (1997), 1779-1783. MR:1397000 (97h:90014)

[16] X. Wu and S. Shen, A further generalization of Yannelis-Prabhakar's continuous selection theorem and its applications, J. Math. Anal. Appl., 197 (1996), 61-74. MF1371276 (97a:47085)

[17] N. C. Yannelis and N. D. Prabhakar, Existence of maximal elements and equilibria in linear topological spaces, J. Math. Economics, 12 (1983), 233-245. MR0743037 (87h:90061a)

[18] Z. T. Yu and L. J. Lin, Continuous selection and fixed point theorems, Nonlinear Anal., 52 (2003), 445-455. MR.1937632 (2003g:54040) 
[19] G. X. Z. Yuan, G. Isac, K. K. Tan, and J. Yu, The study of minimax inequalities, abstract economics and applications to variational inequalities and Nash equilibria, Acta Applicandae Mathematicae, 54 (1998), 135-166. MR1660931 (99j:49018)

[20] X. Zheng, Approximate selection theorems and their applications, J. Math. Anal. Appl., 212 (1997), 88-97. MR1460186 (98h:90118)

Department of Mathematics, National Changhua University of Education, ChangHUA, 50058, TAIWAN

E-mail address: maljlin@math.ncue.edu.tw

Department of Applied Mathematics, National Sun Yat-sen University, and National Center for Theoretical Sciences, Kaohsiung, 80424, Taiwan

E-mail address: wong@math.nsysu.edu.tw

Department of Electrical Engineering, Nan-Kai Institute of Technology, Nantour 542, TAIWAN 